Cahiers d'études africaines

$242 \mid 2021$

Femmes et droit dans les Afriques musulmanes

\title{
FEMINIST AFRICA
}

\section{Sophie Andreetta}

\section{OpenEdition}

\section{Journals}

Édition électronique

URL : https://journals.openedition.org/etudesafricaines/34619

DOI : 10.4000/etudesafricaines.34619

ISSN : $1777-5353$

Éditeur

Éditions de l'EHESS

\section{Édition imprimée}

Date de publication : 15 juin 2021

Pagination : 489-492

ISBN : 9782713228773

ISSN : 0008-0055

Référence électronique

Sophie Andreetta, "FEMINIST AFRICA », Cahiers d'études africaines [En ligne], 242 | 2021, mis en ligne le 15 juin 2021, consulté le 21 avril 2022. URL : http://journals.openedition.org/etudesafricaines/34619 ; DOI : https://doi.org/10.4000/etudesafricaines.34619

Ce document a été généré automatiquement le 21 avril 2022

(c) Cahiers d'Études africaines 


\title{
FEMINIST AFRICA
}

\author{
Sophie Andreetta
}

\section{RÉFÉRENCE}

FEMINIST AFRICA. - Special Issue, « Legal Voice », 15, 2011, 160 p.

1 Articulé autour d'une série d'études de cas, ce numéro spécial vise à fournir aux praticiens une série de pistes de réflexion pour défendre les droits des femmes, en documentant la manière dont, dans certaines réunions ou certaines affaires en particulier, les femmes se sont saisies du droit. Il propose également d'explorer le rapport des femmes à la loi et aux institutions judiciaires sur le continent africain ainsi que le processus de construction des documents juridiques - des conventions internationales aux décisions des tribunaux. Destiné à des juristes, ce numéro spécial permettra néanmoins aux chercheurs en sciences sociales d'identifier des pistes de réflexion, d'envisager des problématiques ou des terrains ethnographiques sur la base des exemples développés - tels que l'accès des femmes mariées à la propriété, la discrimination envers les femmes seules dans les lieux de loisirs ou la manière dont certaines catégories d'acteurs contribuent à l'élaboration des lois.

2 L'introduction au numéro revient avant tout sur la question de la « documentation » en lien avec les expériences et le rapport des femmes au droit. Tamale et Bennet y abordent les difficultés rencontrées par celles et ceux qui utilisent les litiges de manière stratégique pour défendre les droits des femmes en Afrique et le petit nombre de documents sur lesquels ces personnes peuvent s'appuyer : les différentes contributions visent à fournir les outils nécessaires pour que la "voix féministe " soit entendue devant les juridictions. Si la notion de «documentation » n'est que très peu discutée - la littérature sur le sujet n'est pas du tout abordée - Tamale et Bennet explorent cependant la notion de "legal voice " : l'idée que, loin d'être uniformes et identiques, les voix qui mobilisent la loi et saisissent les tribunaux peuvent au contraire être déclinées en fonction du contexte, du genre, de l'orientation sexuelle ou de la couleur de peau des locuteurs (p.9). Les contributions au numéro visent dès lors à documenter le 
processus par lequel des voix féministes deviennent audibles dans le milieu du droit, et ce, de deux manières : à travers des études de cas, souvent basées sur l'expérience professionnelle des auteurs en tant que justiciables, et à travers des articles centrés sur l'adoption d'un texte, la tenue d'une assemblée ou les stratégies d'une organisation en particulier.

3 La première étude de cas retrace l'affaire introduite par Doo Aphane au Swaziland (désormais Eswatini), afin d'obtenir que les biens achetés par des époux sous le régime de la communauté des biens soient également enregistrés au nom de la femme - et non uniquement à celui de l'époux. L'article revient sur les contradictions entre les lois en vigueur depuis plusieurs dizaines d'années et la Constitution, adoptée au début des années 2000 et garantissant l'égalité des sexes - l'argument principal mobilisé par Doo Aphane. Elle obtint gain de cause en première instance, puis en appel: la loi sur l'enregistrement des titres de propriété deviendra alors plus inclusive, permettant aux femmes mariées d'enregistrer des terres à leur nom seul ou avec celui de leur époux. La seconde étude de cas porte sur l'interdiction pour les femmes "non accompagnées » d'entrer dans certains hôtels de Lusaka (Zambie). Après avoir été victime de telles politiques à deux reprises, Sara Hlupekile Longwe saisit le tribunal en mars 1992. L'affaire fut entendue le 30 juillet de la même année - une audience au cours de laquelle le responsable de la sécurité admit que pour éviter la présence de prostituées, l'hôtel interdisait l'accès à ses bâtiments aux femmes seules. Ces articles reviennent cependant très peu sur la littérature sur le foncier en Afrique $^{1}$, sur les dynamiques de genre $^{2}$ ou sur les mobilisations du droit par les femmes dans d'autres contextes. Ils pourraient pourtant apporter une contribution aux débats sur ces problématiques, en mobilisant notamment la jurisprudence, et s'inscrire avec succès dans une réflexion plus large autour des usages du droit en matière de discrimination par exemple ${ }^{3}$, une thématique encore sous-explorée sur le continent africain. Ces articles abordent également des pistes de réflexion empiriques intéressantes en vue de recherches futures : quels sont le rôle, la place et le raisonnement des magistrats dans ces litiges "stratégiques ", dont l'issue peut parfois changer de manière significative le droit et les principes établis?

4 Les autres contributions portent respectivement sur la négociation des droits des personnes LGBTI, des femmes musulmanes et sur le processus d'adoption de la Constitution au Kenya. Sur la base de deux sessions de la Commission africaine sur les droits de l'Homme et des peuples en 2006, Ndashe montre comment les droits des personnes gay, lesbiennes, bisexuelles et transgenres sont négociés à l'échelle internationale, détaillant le travail de lobbying des ONG dont l'objectif est de mettre à l'agenda une série de dispositions, mais aussi d'obtenir le statut d'observateur grâce auquel elles peuvent directement saisir les cours et les tribunaux. Si les travaux autour de la défense des droits fondamentaux au sein d'assemblées internationales sont peu mentionnés dans l'article, le processus concret et les enjeux stratégiques autour du choix des observateurs et de l'obtention de ce statut, pourraient cependant être des pistes de réflexion pertinentes pour les chercheurs en sciences sociales. À travers le cas particulier des femmes musulmanes en Afrique du Sud, Abrahams-Fayker pose la question des droits des femmes au sein de minorités dont les pratiques culturelles spécifiques sont reconnues et protégées. Elle revient sur la question, également abordée dans de nombreux autres contextes, du pluralisme juridique ${ }^{4}$ et de la position des femmes dans la justice islamique. Abrahams-Fayker évoque les dispositifs légaux mis en œuvre dans d'autres pays : une comparaison intéressante, et qui mériterait 
peut-être d'être systématisée à l'ensemble du continent pour réfléchir aux dispositifs possibles et à leurs effets. Enfin, Maingi décrit le travail de FIDA en vue de garantir certains droits pour les femmes dans le cadre de la réforme de la constitution kényane de 2010. Elle décrit les différentes étapes du processus et les points débattus, comme les droits reproductifs des femmes, la définition des groupes ou des personnes vulnérables, l'accès au foncier. Cet article insiste également sur la place des «experts" dans la rédaction de la Constitution - une posture qui pourrait être déconstruite et s'inscrire dans une réflexion plus large sur le parcours, la sélection et le rôle des « experts » dans l'élaboration des lois et des politiques publiques en Afrique.

Ce numéro aborde la question des voix féministes à travers des études de cas empiriques, tantôt fondées sur l'expérience personnelle des auteurs, tantôt sur une revue documentaire d'un processus ou d'un événement en particulier. On y trouvera une série d'exemples discutant les acteurs stratégiques et les enjeux autour du genre dans la construction de la légalité en Afrique. On n'y trouvera pas, en revanche, une discussion de ces études de cas au regard de la littérature existante, ou une réflexion approfondie autour de la manière dont ces voix féministes sont construites et entendues. Si le numéro manque de profondeur analytique, les contributions suscitent néanmoins des questions et permettent d'entrevoir des pistes de réflexion intéressantes en vue de recherches futures - évoquées au fil de ce compte rendu.

\section{NOTES}

1. Voir A. HAUGERUD, "Land Tenure and Agrarian Change in Kenya ", Africa: Journal of the International African Institute, 595 (1), 1989, pp. 61-90 ; S. BERRY, « Debating the Land Question in Africa ", Comparative Studies in Society and History, 44 (4), 2002, pp. 638-668 ; P. PETERS, " "Our Daughters Inherit our Land, but our Sons Use their Wives' Fields": Matrilineal-Matrilocal Land Tenure and the New Land Policy in Malawi ", Journal of Eastern African Studies, 4 (1), 2010, pp. 179-199.

2. P. PETERS, " "Our Daughters Inherit our Land, but our Sons Use their Wives' Fields": Matrilineal-Matrilocal Land Tenure and the New Land Policy in Malawi », op. cit.

3. G. Rosenberg, The Hollowed Hope: Can Courts Bring About Social Change?, Chicago, University of Chicago Press, 1991.

4. A. GRIFFITHS, «The "Women's Question" in Kwena Family Disputes », Journal of Legal Pluralism, 30-31, 1990, pp. 223-254; «Women's Worlds, Siblings in Dispute over Inheritance : A View from Botswana », Africa Today, 49 (1), 2002, pp. 60-82. 


\section{AUTEUR}

SOPHIE ANDREETTA

Laboratoire d'anthropologie sociale et culturelle (LASC), FNRS, Faculté des Sciences sociales,

Université de Liège, Belgique 